Para enlazar con este artículo / To link to this article:

http://dx.doi.org/10.14198/fem.2019.34.04

Para citar este artículo / To cite this article:

Segarra, Marta. «Pour une généalogie de la littérature lesbienne française». En Feminismo/s, 34 (diciembre 2019): 79-96. Dosier monográfico: Estado actual de la investigación en Literatura francesa y Género: balance y nuevas perspectivas / État présent de la recherche en Littérature française et Genre: bilan et nouvelles perspectives, coord. Ángeles Sirvent Ramos, DOI: 10.14198/fem.2019.34.04

\title{
POUR UNE GÉNÉALOGIE DE LA LITTÉRATURE LESBIENNE FRANÇAISE
}

\section{FOR A GENEALOGY OF FRENCH LESBIAN LITERATURE}

\author{
Marta SEGARRA \\ Centre National de la Recherche Scientifique-CNRS, Paris \\ Universitat de Barcelona, Barcelona \\ marta.segarra@cnrs.fr \\ https://orcid.org/0000-0002-1694-962X
}

\section{Résumé}

Ce travail postule qu'une généalogie de la littérature lesbienne française peut être tracée à partir d'un certain nombre d'écrits de femmes des premières décennies du $\mathrm{XX}^{\mathrm{e}}$ siècle, et notamment de la période de l'entre-deux guerres ; parmi ces auteures se trouvent Renée Vivien, Natalie Barney, Lucie Delarue-Mardrus, Jeanne Galzy et Colette. Dans leurs textes poétiques et, dans une moindre mesure, narratifs, l'élément liquide agit souvent en tant que métaphore des relations homoérotiques entre femmes, mais aussi comme image de la fluidité des identifications genrées et sexuelles, au-delà du binarisme homme-femme et hétérosexuel-homosexuel.

Mots-clé: littérature lesbienne française; eau; homoérotisme féminin; Amazones; binarisme sexuel.

\begin{abstract}
This essay posits that a genealogy of French lesbian literature can be traced back to a certain number of women's writings of the first decades of the 20th century, and in particular to the period between the two world wars; among these authors are Renée Vivien, Natalie Barney, Lucie Delarue-Mardrus, Jeanne Galzy and Colette. In their poetic and, to a lesser extent, narrative texts, the liquid element often
\end{abstract}

Los contenidos de la revista se publican bajo una licencia de Creative Commons Reconocimiento 4.0 Internacional (CC BY 4.0)

Feminismo/s 34, diciembre 2019, pp. 79-96 
acts as a metaphor for homoerotic relationships between women, but also as an image of the fluidity of gender and sexual identifications, beyond male-female and heterosexual-homosexual binarism.

Keywords: French lesbian literature; Water; Female homoeroticism; Amazons; Sexual binarism.

\section{UNE LITTÉRATURE LESBIENNE?}

L'émergence de l'écriture des femmes dans la littérature française va de pair, tel que le décrit Anne Emmanuelle Berger dans son article inclus dans ce dossier, avec l'expression de leur désir à la première personne, ou, en d'autres mots, avec l'émergence d'un sujet désirant au féminin qui s'oppose à l'image de la femme en tant qu'objet du désir hétérosexuel. Berger met en rapport le «passage à l'acte littéraire» et le «passage à l'acte homosexuel» chez bien des femmes qui se lancent à l'écriture et à la publication. Cela est bien évident à l'époque du MLF, celle dont s'occupe A. E. Berger dans son article. Mais qu'en est-il des auteures précédentes? J'essaierai ici de poser quelques jalons pour une généalogie de la littérature lesbienne française; il ne s'agira donc pas d'en établir l'histoire, tel que l'a notamment fait Marie-Jo Bonnet, ainsi que d'autres historiennes du mouvement des femmes comme Christine Bard -de façon plus large, dépassant la littérature (Filles de Marianne)-, mais d'évoquer quelques femmes et quelques œuvres singulières qui ont fortement marqué une telle littérature, même si la plupart de ces œuvres sont tombées dans l'oubli. En les sortant du silence critique, il s'agit non seulement de revendiquer leur intérêt pour le public lecteur d'aujourd'hui, mais aussi de s'opposer au récit dominant de la littérature française du $\mathrm{XX}^{\mathrm{e}}$ siècle, qui a exclu ces voix -ou voies- dissidentes ${ }^{1}$.

Il faudrait quand même éclaircir au préalable ce que j'entends par littérature lesbienne. Nous pourrions partir d'une conception large du terme «lesbienne», comme celle d'Adrienne Rich, qui a proposé la notion d'«existence

1. Cet article trouve son origine dans un travail entamé avec Anne Emmanuelle Berger sur l'histoire culturelle des femmes et de la littérature du XXe et XXI siècles. Il a été exposé en partie lors de la conférence inaugurale du XXV Colloque AFUE tenu à l'université Politècnica de València le 20 avril 2016. 
lesbienne» et de «continuum lesbien» pour couvrir un large éventail de relations que les femmes établissent entre elles en tant qu'actes de «résistance au patriarcat», des relations qu'on pourrait aussi qualifier d'homo-affectives ou même d'homosociales. Rich postule ainsi qu'on peut observer au long de l'histoire occidentale toute une série de stratégies de résistance à l'oppression patriarcale, en rapport avec la sexualité, déployées par des femmes allant de Sappho et son école de poésie aux béguines, passant par les précieuses et leur méfiance envers le mariage en tant qu'instrument de domination masculine et arrivant jusqu'aux féministes contemporaines; ces femmes se sont efforcées de mener ce que Rich appelle une «existence lesbienne». Je m'intéresserai aussi ici aux théorisations de Luce Irigaray par rapport à l'«homosexualité primaire» qui serait partagée par toutes les femmes, puisque le premier «corps à corps» que toute femme aurait connu, selon Irigaray, serait celui qui se produit avec le corps de la mère.

Définir ce que serait la «littérature lesbienne» est donc difficile, d'autant plus que, comme l'a montré Foucault (Histoire de la sexualité), ce que ce terme recoupe est un effet de discours et que ces catégories ne peuvent pas être utilisées de façon anhistorique. Il n'est pas non plus pertinent, à mon avis, de se baser sur les mœurs affectives ou sexuelles des auteures -ce qui conduirait, par exemple, au contresens d'inclure les romans de Marguerite Yourcenar dans la «littérature lesbienne». De façon bien plus intéressante, Monique Wittig signale que le début de la «littérature lesbienne» se trouve chez Marcel Proust et Djuna Barnes, c'est-à-dire entre les années vingt et trente du XXe siècle, puisqu'elle considère que ces deux auteurs sont les premiers à avoir fait de la lesbienne et du «genre féminin»-Wittig utilise ici le terme «genre» dans son sens grammatical - «l'axe de catégorisation à partir duquel universaliser» (Pensée straight 91). En d'autres mots, À la recherche du temps perdu et Le Bois de la nuit de Barnes sont selon Wittig des cuvres «stratégique[s]» par leur «réalité textuelle» et non par leur focalisation sur un «thème social» (91); c'est bien parce que ce sont des «œuvre[s] littéraire[s] importante[s]» que ces textes réussissent à «rendre universel le point de vue minoritaire» (92).

Ma définition de la «littérature lesbienne» dans cet article serait ainsi, simplement, la suivante: j'entends par ce terme un corpus d'œuvres, écrites en général par des femmes mais parfois aussi par des hommes, qui se focalisent sur les relations affectives et homoérotiques entre femmes autant sur le plan

Feminismo/s 34, diciembre 2019, pp. 79-96 
du contenu (le «thème social» dont parle Wittig) que sur celui de la forme (car ces œuvres inventent de nouvelles façons de présenter cette thématique). Lécriture du désir de la part des femmes dépasse, dès son apparition dans la littérature française, le cadre hétérosexuel; néanmoins, de la même façon que les romans jugés «érotiques» écrits par des femmes laissent parfois penser que leur but est de faire plaisir aux hommes plutôt que d'inscrire un désir qui serait spécifique aux femmes, l'érotisme lesbien a traditionnellement fait partie des fantasmes des hommes hétérosexuels; la série des Claudine de Colette, par exemple, pourrait ainsi être considérée comme appartenant à cette littérature censée être «lesbienne» mais en réalité destinée à émoustiller les lecteurs hétérosexuels. D’autres ouvrages, notamment au XIX siècle, mettent en scène l'«invertie» ou la «lesbienne criminelle», en tant que figure marginale et dangereuse (tel que l'ont étudié, entre autres, Jennifer WaeltiWalters ou Christopher Robinson): venant de plumes d'hommes mais aussi de femmes comme Rachilde, souvent inspirés par les discours médicaux qui décrivaient l'«homosexualité» tout en la créant, ces textes stigmatisent les individus concernés et font de la lesbienne l'incarnation de la sexualité féminine débridée et donc menaçant l'ordre social (Marks).

Je ne tiendrai pas compte ici, en tant que «littérature lesbienne», de ces textes qui mettent en scène cette figure de la «lesbienne criminelle», ainsi que des textes-fraude faisant semblant de s'occuper des relations entre femmes mais où ce thème est en réalité une excuse pour relever le récit, destiné à un public «normal», à des fins érotiques; j'en exclus enfin des textes qui mettent en scène des personnages que nous pourrions considérer comme des lesbiennes mais qui ne touchent pas à leurs rapports affectifs et sexuels avec d'autres femmes.

Quel serait donc l'intérêt d'établir une généalogie de la «littérature lesbienne» dans ce sens que je viens d'expliquer? En premier lieu, il y aurait bien sûr un aspect historique, sociologique et politique à considérer, concernant les formes que prend la constitution de l'objet «lesbienne» et «lesbianisme» dans l'histoire culturelle et littéraire française. Une autre facette intéressante relève cependant de la nature spécifiquement littéraire des textes que j'évoquerai ici, c'est-à-dire de leur forme, autant en ce qui concerne la langue que les structures narratives ou encore l'inscription de la subjectivité dans le texte. En effet, je postulerai, exemples à l'appui, qu'au moment de la constitution

Feminismo/s 34, diciembre 2019, pp. 79-96 
de l'objet «littérature lesbienne»-que je situerai, suivant Wittig, essentiellement entre les années vingt et quarante du $\mathrm{XX}^{\mathrm{e}}$ siècle, c'est-à-dire la période de l'entre-deux-guerres-, des traits communs peuvent être observés dans les textes compris dans ce champ; ce sont des traits qui comprennent, sinon une allusion explicite à l'eau en tant que métaphore des relations homoérotiques féminines, au moins une représentation de celles-ci, ainsi que des corps des femmes impliquées, qui souligne leur fluidité, leur «liquidité». Charles Maurras y fait allusion dans son essai Le Romantisme féminin: «Les vers d'Ondine ne sont-ils pas liquides, onctueux et charnels jusqu'au point de faire sentir les sinuosités d'un corps tiède et lascif?» (182). Au-delà de la vision de la féminité stéréotypée et voyeuriste de ce critique, il m'importe de remarquer son évocation de la forme de ce poème de Renée Vivien.

\section{LES AMANTES MARINES}

Le premier moment de la littérature «lesbienne» en France -terme auquel l'époque préférait plutôt «saphique» ou «tribade», des mots évoquant la Grèce antique, «tribade» se référant à une pratique sexuelle et «saphique» étant une allusion littéraire à Sappho- procèderait donc du cercle des «Amazones» et autres lesbiennes notoires du Paris de la Belle Époque, aussi appelé «ParisLesbos» (Bonnet; Waelti-Walters). Cette époque marque un moment de tolérance envers cette «déviance» de l'hétérosexualité normative; les femmes qui aimaient d'autres femmes avaient ainsi leurs lieux de socialisation, comme le café Le Monocle, où le public était en partie composé de femmes travesties en hommes et où cet objet agissait comme signe d'appartenance et de reconnaissance.

La plupart des écrivaines et des artistes qui composaient le cercle des Amazones étaient d'origine non française (procédant surtout des États-Unis et d'autres pays européens) et écrivaient souvent dans leur langue maternelle et non en français, ce qui les exclut de notre généalogie. Se trouvaient parmi elles Gertrude Stein et sa compagne Alice Toklas; M. Radclyffe Hall, l'auteure de ce qui est jugé le premier roman «lesbien» de la littérature états-unienne, Le Puits de solitude, publié en 1928; des artistes comme Romaine Brooks, des éditrices et libraires comme Sylvia Beach et Adrienne Monnier,... L'essai de Shari Benstock ainsi que le livre et le film d'Andrea Weiss font le récit de 
cette époque dorée pour ces premières femmes échappées -parfois pour un moment seulement- du seul rôle qui était permis aux femmes de leur classe sociale, celui d'épouse et mère.

La figure autour de laquelle tournait le cercle des Amazones est celle de Natalie Clifford Barney, riche héritière d'origine états-unienne qui s'installe très jeune en France. Barney, auteure de plusieurs recueils de poèmes et de textes narratifs en français, est l'amie d'une autre poète d'origine britannique mais écrivant en français, Renée Vivien, qui a une œuvre courte puisqu'elle mourut très jeune, mais dont la poésie est plus intéressante que celle de Barney pour mon propos. Le recueil de Renée Vivien Études et préludes, auquel faisait référence Maurras, offre, dès la «Dédicace», une représentation du corps féminin en tant qu'élément liquide: "Ton corps se devinait, ondoiement incertain / Plus souple que la vague et plus frais que l'écume» (3). Et, de façon plus subtile et détournée, dans: «Parmi des flots de sons languissamment décrus, / Blonde, tu m'apparus» (4). Ces deux derniers vers, bien que se référant apparemment à des éléments sonores et non liquides, dessinent une sorte d'apparition mythique, comme celle traditionnelle de Vénus surgissant de la mer, par l'usage des mots «flots» et «décrus», qui s'apparentent normalement à l'eau. Le poème cité par Maurras, «Ondine», inclut les vers suivants:

Ton rire est clair, ta caresse est profonde,

Tes froids baisers aiment le mal qu'ils font;

Tes yeux sont bleus comme un lotus sur l'onde

Et les lys d'eau sont moins purs que ton front.

Ta forme fuit, ta démarche est fluide,

Et tes cheveux sont de légers réseaux ;

Ta voix ruisselle ainsi qu'un flot perfide

Tes souples bras sont pareils aux roseaux,

Aux longs roseaux des fleuves, dont l'étreinte

Enlace, étouffe, étrangle savamment,

$\mathrm{Au}$ fond des flots, une agonie éteinte

Dans un nocturne évanouissement. (39-40)

Dans ce fragment, tout un réseau sémantique fait allusion à l'eau («clair», «froids», «bleu», «lotus», «onde», «lys d'eau», «pur», «fluide», «ruisselle», «flot», «roseaux»-deux fois-, «fleuves», «flot»), mais le commentaire de Maurras semble toucher plutôt à la construction même des vers, qui 
reproduirait en quelque sorte l'ondoiement liquide du corps féminin aimé. Il est superflu de rappeler l'association bien connue entre l'eau et la féminité -que Bachelard a longuement analysé-, mais je soulignerai ici la relation entre le corps féminin, ainsi que la féminité en général, et l'inclinaison du corps, par opposition à la verticalité du corps masculin et de la masculinité. Une des réflexions récentes les plus suggestives à cet égard est celle de la philosophe italienne Adriana Cavarero, qui met en rapport ce corps incliné avec le concept de vulnérabilité que Judith Butler a développé (Saez Tajafuerce). Par ailleurs, dans «Ondine» de Vivien, l'évocation de l'eau se fait aussi à travers les assonances mises en œuvre dans le texte, qui thématise, comme dans la «Dédicace», l'association entre le son et l'élément liquide.

Lucie Delarue-Mardrus, contemporaine de Renée Vivien, écrivit aussi des poèmes surgis de sa liaison avec Natalie Barney, des textes publiés posthumément par les soins de celle-ci dans un recueil intitulé Nos secrètes amours (1951). Dans ces poèmes, Delarue-Mardrus a souvent recours à des images aquatiques pour se référer à celle qu'elle appelle son «amante marine»: «Sous ma bouche salée encore par la mer, / Sa bouche / Est humide et glissante comme de l'eau douce, / Et telle est la blessure intime de sa chair» (2). Ces vers sont plus osés que ceux de Vivien puisqu'ils font allusion à des pratiques sexuelles; mais c'est le contraste entre l'image de l'eau salée et de l'eau douce qui est original et riche, dans le sens qu'il brouille en quelque sorte la gémellité, souvent rendue par l'image du miroir, qui réduit -non seulement à cette époque- l'homosexualité et spécialement le lesbianisme à «du même», à un refus ou un déni de la relation à l'Autre que signifierait l'amour hétérosexuel par rapport à l'homosexuel. Cette vision de l'homosexualité revient en fait à nier l'altérité de l'autre aimé en tant qu'individu, voyant cet autre simplement comme une personne classifiée dans tel ou tel «sexe» ou «genre»...

Dans le poème «Pour toi» du même recueil de Lucie Delarue-Mardrus, le sujet poétique se compare à la mer: «Toujours je me transforme et suis toujours la même / Comme la mer multiple et une d'où je sors, / Qui recule et se rue à jamais et quand même / Vers la possession des villes et des ports / Et des terres avec leurs prés et leurs bois tors / Qu'Elle n'atteindra pas de son spasme suprême» (2).

Le deuxième vers du fragment cité, "Comme la mer multiple et une d'où je sors», nous rappelle que l'homophonie en français de mère et mer renforce 
puissamment cet imaginaire, très généralisé, qui associe la mer ou l'élément liquide à la féminité maternelle. La sexualisation de la mer/mère (avec l'allusion à «son spasme suprême») peut de même être mise en rapport avec ce que Luce Irigaray appelle «lesbianisme primaire», qui serait celui du «corpsà-corps avec la mère»; j'y reviendrai.

Cette citation montre bien la conjonction entre la thématisation de l'image de l'eau ou de la liquidité, et la façon dont celle-ci est rendue textuellement; la construction syntaxique et lexicale des vers de «Pour toi» reproduit le mouvement ondoyant, d'aller et retour des vagues: «Toujours je me transforme et suis toujours la même», "Qui recule et se rue à jamais et quand même»); les assonances et la rime en «m» renvoient également à l'élément marin. Dans un autre recueil, Delarue-Mardrus utilise l'image liquide de façon encore plus choquante pour l'époque; Maurras n'ose même pas citer dans son essai les vers suivants, y faisant référence de façon seulement indirecte en les qualifiant de «pervers» par leur refus de la maternité (Engelkin): «Et, parmi mes coussins pleins d'ombre, je m'enivre / De ma stérilité qui saigne lentement» (Delarue-Mardrus, Horizons 176-177). Il n'est donc pas nécessaire d'attendre les auteures des «années MLF» comme Viviane Forrester ${ }^{2}$ pour assister à l'entrée du sang menstruel dans la poésie française...

\section{LES AMAZONES SURGISSANT DES FLOTS}

Suivant la définition de «littérature lesbienne» en tant que celle qui thématise et «universalise», dans les termes de Monique Wittig, les rapports homoérotiques entre femmes, il faut aussi s'intéresser à des ouvrages narratifs, bien qu'il soit plus difficile d'y percevoir une textualisation de l'élément liquide. Renée Vivien publie en 1904 le roman Une femme m'apparût, qui met en scène sa rencontre et sa relation avec Natalie Barney. Ce qui intéresse mon propos, c'est que l'auteure inscrit leur rapport lesbien dans une réaction contre la domination masculine:

Or les actions des hommes ont toujours eu pour but unique l'asservissement de la femme à leur caprice stupide, à leur sensualité, à leur tyrannie injuste et féroce. Et comment ne point haïr un individu qui se présente à vous sous

2. Voir l'article d'Anne Emmanuelle Berger dans ce même dossier.

Feminismo/s 34, diciembre 2019, pp. 79-96 
les espèces d'un maître? Tout être intelligent et fier se révolte nécessairement contre le joug d'un autre être, parfois son égal mais souvent son inférieur. (103; cité dans Bonnet 256)

Natalie Barney se montre, de son côté, encore plus radicale dans son exclusion des hommes: «Il est temps que les Amazones ne se fassent plus féconder par 'l'ennemi', et l'ennemi n'est-il pas celui qui prendra à la femme son enfant, pour l'élever ou le tuer à sa guise?» (9) -retenons l'allusion à la relation mère-enfant, à laquelle je reviendrai par rapport au lesbianisme.

Ces deux citations font fortement écho à des raisonnements que fera bien plus tard une lesbienne radicale comme Monique Wittig, quand elle affirme de façon polémique que seule la lesbienne échappe à ce qu'elle appelle la «pensée straight» ou à la matrice hétérosexuelle des rapports sociaux et intimes, puisque les «femmes» sont toujours définies en rapport avec les hommes. Wittig ouvre ainsi la porte à la théorie queer, en montrant une voie par où échapper à la binarité du sexe et du genre, mais établit aussi une différence controversée entre les lesbiennes «politiques» et les femmes aimant d'autres femmes ${ }^{3}$. Rappelons aussi que Wittig est non seulement l'auteure du Corps lesbien ${ }^{4}$, mais également du roman épique Les Guérillères où elle récupère le mythe des Amazones, renouant donc avec ces précurseures de la Belle Époque.

Le roman de Colette Le Pur et l'Impur (publié en 1932 dans une première version intitulée Ces plaisirs... et dans une version corrigée, sous ce nouveau titre, en 1941) et celui de Lucie Delarue-Mardrus L'Ange et les Pervers (1934) sont des témoignages fictionnalisés de leur propre participation amicale au cercle lesbien du Paris de la Belle Époque, écrits des années après leur fréquentation de Natalie Barney et ses amies. L'«ange» du titre du roman de DelarueMardrus, un alter ego de l'auteure mais aussi inspiré de Renée Vivien, aurait grandi jusqu'à dix ans se croyant un garçon, pour se découvrir femme à la puberté. Ce personnage porte un prénom ambigu (Marion/Mario) et se considère comme un «homme de lettres», à l'image des «bas-bleus» du XIX siècle. En réalité, ilelle juge son genre «neutre»-c'est pourquoi il faut un nouveau

\footnotetext{
3. Voir le travail d'Ilana Eloit à ce sujet concernant Wittig et d'autres féministes françaises et états-uniennes des «années MLF».

4. Voir le commentaire qu'en fait Anne Emmanuelle Berger dans son article.
}

Feminismo/s 34, diciembre 2019, pp. 79-96 
pronom pour le désigner, comme ceux que proposera quelques décennies plus tard Monique Wittig dans ses textes de fiction. L'œuvre de Lucie DelarueMardrus avait toujours oscillé entre le chant de la sensualité, notamment homoérotique, et l'attirance envers la «virginité», dans un sens similaire à celui de la féministe, qui lui était contemporaine, Madeleine Pelletier, auteure d'un roman autobiographique intitulé La Femme vierge (1933) où la protagoniste décide de renoncer à la sexualité puisque ses conséquences sont trop terribles pour les femmes (une de ses amies a failli mourir d'un avortement clandestin); dans L'Ange et les Pervers, de même, le personnage incarnant Natalie Barney rétorque à une ancienne maîtresse qui se découvre enceinte tout en étant séparée de son mari et se plaint d'avoir été débauchée par l'aristocrate: «Nos histoires ne t'ont jamais fait d'enfant !...» (99).

Ces personnages comme ces femmes réelles -dont Madeleine Pelletier-se refusent ainsi à entrer dans ce que cette féministe appelle «le marché du sexe», s'abstenant pour certaines de s'habiller comme les femmes de leur époque et ne portant que des tailleurs masculins. Colette-l'antithèse de ce qui pourrait être appelé anachroniquement la «lesbienne politique»- ne pourra pas être d'accord avec ce refus de la «féminité», qu'elle défend ardemment, et dans Le Pur et l'Impur se trouvent des commentaires cinglants sur les femmes qui «singent l'homme» en lui empruntant ses habits et ses manières, de façon peu réussie selon Colette (oubliant cependant qu'elle avait aussi joué de l'ambiguïté du travestissement et, surtout, qu'elle avait aimé une femme, Mathilde de Morny, qui s'habillait généralement en homme). Colette considère l'homosexualité féminine comme une gémellité agissant en tant que repaire provisoire face aux souffrances de l'amour hétérosexuel, comme un havre de paix temporaire. Mais elle dit aussi: «La séduction qui émane d'un être au sexe incertain ou dissimulé est puissante» (596) -phrase qui incite à queeriser Colette...

En même temps, l'ambivalence des amours racontées par Colette, dans la série romanesque autour de Chéri notamment, entre un jeune homme et une femme bien plus âgée que lui, prend des tonalités clairement incestueuses; malgré leur nature apparemment bien hétérosexuelle, ces récits peuvent donc être rapprochés du lesbianisme "primaire», par ces allusions transparentes aux relations mère-enfant et par la féminisation du jeune amant, parfois à peine sorti de l'enfance. De même, le roman Carnaval (1923) de Mireille

Feminismo/s 34, diciembre 2019, pp. 79-96 
Havet, auteure ouvertement lesbienne ${ }^{5}$, contient cette phrase révélatrice, dite à son jeune amant par une femme mûre: «Tu es mon enfant. Toute ma chair te reconnaît, toute ma chair te désire» (59), phrase qui ramène un amour apparemment hétérosexuel au «lesbianisme primaire» d'Irigaray.

La fin de L'Ange et les Pervers, le roman de Delarue-Mardrus, a été généralement jugée par la critique comme une clôture consensuelle et moralisatrice d'une histoire sulfureuse, où tout rentre dans l'ordre puisque Marion/Mario abandonne sa double vie en tant que femme et homme (renonciation matérialisée par le geste symbolique de quitter ses deux appartements, l'un rive gauche où elle vit comme une femme, l'autre rive droite où elle se produit en homme) quand elle se découvre des sentiments maternels pour le petit garçon bâtard abandonné par sa mère et décide de l'adopter. Cependant, ce retournement rapide de l'intrigue peut être interprété autrement, car Marion/ Mario ne renie pas son côté masculin: «Mon amour maternel sera toujours un peu mâle, forcément [...] Aux yeux de beaucoup, je vais passer pour une fille-mère. Quelle gloire, quand on est un œuf clair! [...] Je serai son père et sa mère en une seule personne... J'ai donc raison d'être deux» (156).

Ces phrases finales nous montrent qu'ilelle ne renonce pas à son double genre/sexe (qu'elle désigne par l'expression «œuf clair», se référant à son refus d'avoir des relations sexuelles, avec des hommes comme avec des femmes) et que cet enfant, qui est aussi décrit sous des traits féminisés tout en étant un garçon, sera enfin son vrai partenaire lesbien, dans le sens d'Irigaray. En même temps, cette assomption finale du double sexe/genre-le personnage se définit comme un «monstre de conte de fées» (42) et un «sphinx» (34), image reprise un demi-siècle après dans le roman marquant d'Anne F. Garréta- équivaut bel et bien à son refus d'entrer dans la «matrice hétérosexuelle» wittigienne, puisqu'elle se libère enfin d'avoir été «rang[é.e] du côté de la régularité bourgeoise» (48) par le médecin qui l'a examiné.e lors de sa puberté: «Celui-ci déclara, malgré mon état-civil, qu'à tout prendre il était préférable de me cataloguer dans le sexe féminin» (46). Michel Foucault reprendra une histoire similaire, mais réelle, celle d'Herculine Barbin, aussi obligé.e de se «ranger»

5. Havet décrit dans son journal, en 1918, combien elle déteste -physiquement- les hommes. La jeune femme se réclame du cercle des Amazones, et notamment de N. Barney, R. Vivien et Colette, pour justifier ses préférences homosociales et homosexuelles.

Feminismo/s 34, diciembre 2019, pp. 79-96 
dans un sexe-genre par les autorités civiles et religieuses; il n'est pas banal non plus que ce sexe-genre choisi un peu arbitrairement soit le féminin, celui qui marque la «différence» par rapport à la «norme».

Ces cas concernant le «neutre», la «vierge», l'«amazone», l'«ange» ou enfin la «lesbienne» comme «troisième sexe»-formule dont Willy, le premier mari de Colette, assurera la fortune-, peuvent être mis en rapport avec un des exemples, de la même époque, les plus riches et originaux concernant la mise en scène de la différence des sexes et de la confusion et la multiplication des genres, celui des portraits de Claude Cahun. Photographe de génie aujourd'hui jugée précurseure de l'indéfinition sexuelle et de son jeu infini de variantes, Cahun est à l'opposé du binarisme masculin/féminin, notamment par ses autoportraits très singuliers où elle se représente en homme, femme, androgyne ou même en animal, jouant souvent avec son double au miroir. Les écrits de Cahun, moins connus que ses photographies, placent son œuvre écrite dans la tradition de la littérature des femmes par ses textes sur des consœurs «célèbres»: le recueil inachevé Héroinnes (1925) fait ainsi le portrait, entre autres, d'Ève, Dalila, Judith («la sadique»), Pénélope, «Hélène la rebelle» ou «Sapho l'incomprise». Héroïnes inclut une réécriture de l'histoire de Cendrillon, «Cendrillon, l'enfant humble et hautaine», qui met en valeur l'indépendance et l'agentivité du personnage, son masochisme volontairement assumé mais aussi son rôle de «maîtresse hautaine, dominatrice, aux talons durs et sans pitié» (143), rôle qu'elle revêt pour séduire le prince adepte du fétichisme. C'est une «Cendrillon blasée» qui, en faisant la rencontre du prince, s'exclame: «Et peut-être l'aimerais-je sincèrement s'il voulait quelquefois invertir nos rôles... Il n'y faut point songer: si je gâtais ses illusions, bien vite il renverrait le grillon au foyer! -Le tromper jusqu'à la tombe» (144). Cette Cendrillon préfigure aussi l'importance que les pratiques sexuelles non-normatives auront dans la théorie et l'art féministes contemporains. Dans ce recueil de contes inachevé de Cahun, se détache également une version postmoderne de «La Belle»: "Quand on a goûté de la Bête -ah! que l'homme est chère fade» (150)- la confusion chère-chair pouvant être autant une coquille qu'un jeu de mots. Cahun reprend les contes de fées en les rendant ouvertement féministes; elle affirme ainsi: «Le féminisme est déjà dans les fées» (234). Mais au lieu de renverser simplement les rôles des personnages comme dans une certaine littérature de l'époque du MLF qui essayait

Feminismo/s 34, diciembre 2019, pp. 79-96 
de récrire les contes populaires en les dépouillant de leur misogynie, Cahun les rend bien plus complexes. L'animalisation des personnages, fréquente dans ces contes, devient une façon de rendre explicite le flou identitaire, les passages, les identifications qui tiennent lieu d'identité.

Dans le même souci de représentation de soi que celui manifesté dans ses autoportraits, Scrap book, volume recueillant les notes de Cahun prises pendant la Deuxième Guerre mondiale, dénonce déjà, bien avant Foucault ou Deleuze et Guattari, les «machines légales, psychiatriques ou policières, machines armées de techniques efficaces» (656) destinées à dompter les personnalités rebelles, comme celle de l'auteure, et les soumettre au formatage de la pensée straight. Un détail révélateur de sa subtilité est la façon dont elle raconte sa convocation au bureau de la Gestapo. Cahun et sa compagne Moore s'étaient risquées à résister sournoisement -mais aussi dangereusement que les résistants qui posaient des bombes- à l'ennemi allemand sur l'île de Jersey où elles habitaient à l'époque de la Deuxième Guerre mondiale. Pour les tromper sur son identité, l'auteure alla trouver les autorités de l'occupant se rendant «méconnaissable -en Lucy Schwob» (son nom de naissance), tandis qu'elle «vivai[t] normalement sous [s] on aspect Claude Cahun» (630).

Ce jeu avec le binarisme du sexe/genre avait déjà été entamé par la popularité de la «garçonne» (Bard, Garçonnes), terme procédant du roman de Victor Margueritte publié en 1923. La «garçonne», modèle de femme qui résume les changements dans la définition de la «féminité» après la Grande Guerre, est aussi perçue comme un «troisième sexe» menaçant la suprématie masculine, un renversement du pouvoir qui rend l'homme soumis à la femme toute-puissante ${ }^{6}$. Ainsi, dans Le Pur et l'Impur de Colette, la narratrice avoue que les hommes la fuient à cause de son «hermaphrodisme mental» (idée typique de l'époque, exprimée aussi par Delarue-Mardrus), et qu'elle aurait voulu sacrifier cette «virilité» en la jetant «aux pieds d'un homme» et offrant à celui-ci «un brave corps bien femelle et sa vocation, peut-être fallacieuse, de servante» (586). Malgré cette expression du désir de soumission à l'homme,

6. Le «coming out» de Mireille Havet en tant que lesbienne-véritable «épiphanie identitaire», dit sa biographie-s'effectue en 1917, grâce à une coupe de cheveux à la garçonne; elle en parle dans son journal comme d'une vraie «libération». À l'époque, cette coupe à la garçonne ne s'étant pas encore généralisée, la nuque rasée est considérée comme un signe de lesbianisme (Retaillaud-Bajac 198).

Feminismo/s 34, diciembre 2019, pp. 79-96 
tout à fait contraire aux remarques de Vivien et de Barney au même propos, la narratrice de Colette spécifie bien que les hommes la fuient parce que «certaines femmes représentent, pour certains hommes, un danger d'homosexualité» (587). Seules des consœurs peuvent apprécier la «virilité spirituelle» de ce type de femmes, conclut-elle, ce qui nous ramène au lesbianisme et à la fluidité des genres.

Les rapports mère-fille que Colette décrit à travers le personnage de Sido pourraient aussi être rapportés à ce «lesbianisme primaire» dont se réclament implicitement les écrivaines citées; cependant, les textes de Colette seraient, si on suit Kristeva (20), plutôt un «hymne», aux échos «paiens», athées et amoraux, à une «jouissance» jugée spécifique aux femmes, qui déborderait la sexualité pour s'étendre sur les sensations et les plaisirs apportés par tous les sens, allant du contact avec les plantes apporté par le jardinage jusqu'à la gourmandise (Dupont). Cette revendication de la jouissance rapprocherait d'ailleurs Colette de ses héritières du MLF, bien plus que de la plupart des féministes, plutôt rangées et même prudes, de son temps. Cependant, la passion pour la jouissance enchaîne autant qu'elle déchaîne, et Colette décrit aussi vivement les servitudes et les extases de «ces plaisirs qu'on nomme, à la légère, physiques» (citée dans Kristeva 399), mais qui atteignent néanmoins pour elle une dimension «cosmique».

Bien moins connue aujourd'hui mais ayant joui d'un grand succès à l'époque, Jeanne Galzy écrit des romans focalisés sur l'homoérotisme féminin, à peine déguisé. Lauteure situe ces relations entre femmes dans des lieux fermés, des «hétérotopies» dans le sens foucaldien: l'école normale de Sèvres (réservée aux filles, de futures institutrices) dans son roman Jeunes filles en serre chaude (1934) et un sanatorium pour malades de tuberculose (Les Allongés, 1923). Ce dernier ouvrage est dans la tradition des grands romans «tuberculeux» mais, parmi les effets de la maladie qui y sont décrits, il est intéressant de remarquer la liquéfaction des organes du corps (conduisant à une sorte de «corps sans organes» deleuzien), ainsi que la confusion des genres: les «allongés» perdent leurs traits sexués différentiels, subissent une sorte de féminisation généralisée (en rapport avec leur position horizontale, tel que le titre le suggère) et maintiennent entre eux des rapports ambigument sensuels. Jeunes filles en serre chaude, de son côté, reprend la tradition de l'amour comme éducation de la jeune aimée pour la subvertir, puisque

Feminismo/s 34, diciembre 2019, pp. 79-96 
les relations établies dans le roman entre la protagoniste, une étudiante de l'école, et sa professeure d'anglais ne sont pas hiérarchiques et n'aboutissent pas à une initiation ou même à un apprentissage de la jeune femme, si ce n'est la prise en main de sa propre vie affective et sexuelle: la protagoniste connaît «l'orgueil de s'admettre soi-même avec tous ses actes, toutes ses possibilités d'actes, d'accepter quelque chose qui était un accomplissement, de s'engager envers son avenir» (113). Létudiante est finalement mise sur le même plan que son aînée, puisque les deux se sentent également attirées par un jeune individu de prénom et de genre ambigu. Le roman dérive ainsi vers l'ambiguité, la multiplication ou la «chorégraphie» des genres, selon le mot de Derrida. Une fête à l'école où toutes les étudiantes se déguisent sert ainsi de prétexte à des travestissements révélateurs; la femme la plus «laide» se retrouve admirée en Hamlet: «Fluide éphèbe, jeune homme de songe -et non plus jeune fille sèche» (104).

\section{POUR CONCLURE}

Le fil rouge de l'eau en tant que métaphore des relations homoérotiques entre femmes est moins visible dans les ouvrages narratifs, notamment sur le plan de la construction formelle ou textuelle. Une exception significative serait $L e$ Pur et l'Impur de Colette, qui se termine par une évocation du signifiant «pur», prononcé par une femme évoquant son amour parfait pour une autre femme :

De ce mot pur qui tombait de sa bouche, j'ai écouté le tremblement bref, l'u plaintif, l'r de glace limpide. Il n'éveillait rien en moi, sauf le besoin d'entendre encore sa résonance unique, son écho de goutte qui sourd, se détache et rejoint une eau invisible. Le mot "pur» ne m'a pas découvert son sens intelligible. Je n'en suis qu'à étancher une soif optique de pureté dans les transparences qui l'évoquent, dans les bulles, l'eau massive, et les sites imaginaires retranchés, hors d'atteinte, au sein d'un épais cristal. (653)

L'écriture magistrale de Colette réussit à combiner ici l'évocation sémantique et l'inscription textuelle de l'eau dans cette allusion au désir lesbien, exprimée par le son du mot «pur» mais où tous les sens sont convoqués, confirmant cette ambition totalisante de l'écrivaine dans la représentation d'un désir et d'une jouissance qui seraient pour elle spécifiquement féminins -mais pas exclusivement réservés aux «femmes», tel qu'on l'a vu, ce qui pourrait 
convenir aussi à caractériser une des écrivaines et théoriciennes du MLF, Hélène Cixous.

Cependant, mon propos en convoquant ces romans était plutôt de montrer comment ceux-ci inscrivent dans le récit, bien avant l'avènement de la pensée queer, une fluidification des identités, notamment sexuelles et genrées, qui deviennent liquides, ondoyantes, changeantes et donc irréductibles à la loi binaire de l'hétérosexualité. Dans ces textes, l'élément liquide n'est pas tant une métaphore pour signifier «le corps lesbien», pour le dire avec Monique Wittig, qu'une image du brouillage des genres, des sexes et des corps en tant qu'effet du désir lesbien.

\section{RÉFÉRENCES BIBLIOGRAPHIQUES}

Bachelard, Gaston. L'Eau et les Rêves. Essai sur l'imagination de la matière. Paris: José Corti, 1942.

Bard, Christine. Les Filles de Marianne. Histoire des féminismes (1914-1940). Paris: Fayard, 1995.

Bard, Christine. Les Garçonnes. Mode et fantasmes des Années folles. Paris: Flammarion, 1998.

Barney, Natalie. Pensées d'une Amazone, Paris: Émile Paul, 1918.

Benstock, Shari. Femmes de la Rive gauche: Paris, 1900-1940. Paris: Éditions des femmes, 1987.

Bonnet, Marie-Jo. Les Relations amoureuses entre les femmes du XVI au XXe siècle. Paris: Odile Jacob, 1995 (nouv. éd. revue et corr.).

Cahun, Claude. Écrits. Éd. François Leperlier. Paris: Jean-Michel Place, 2002.

Colette. Le Pur et l'Impur. Euvres. Vol. 3. Éd. Claude Pichois. Paris: GallimardNRF, «Bibliothèque de la Pléiade», 1991.

Delarue-Mardrus, Lucie. Horizons. Paris: Fasquelle, 1904.

Delarue-Mardrus, Lucie. L'Ange et les Pervers. Paris: Ferenczi, 1932.

Delarue-Mardrus, Lucie. Nos secrètes amours. Paris: les Isles, 1951.

Deleuze, Gilles, et Félix Guattari. L'Anti-CEdipe. Capitalisme et schizophrénie I. Paris: Les Éditions de Minuit, 1972.

Derrida, Jacques, et Christie McDonald. «Chorégraphies». Points de suspension. Entretiens. Jacques Derrida. Éd. Élisabeth Weber. Paris: Galilée, 1992. 95-115. Dupont, Jacques. Physique de Colette. Toulouse: Presses Universitaires du Mirail, 2003. 
Eloit, Ilana. «Heterosexual French Feminism and the Americanization of Lesbianism in the 1970's». Feminist Theory. 2019. (Sous presse)

Engelkin, Tama L. «LAnge et les Pervers: Lucie Delarue-Mardrus' Ambivalent Poetic Identity». EngagedScolarship@CSU. 11-1992. http://engagedscholarship.csuohio.edu/cgi/viewcontent.cgi?article $=1027$ \&amp;context=clmlang_facpub

Foucault, Michel. Histoire de la sexualité I. La volonté de savoir. Paris: Gallimard, 1976.

Foucault, Michel. Les Hétérotopies. Le Corps utopique. Paris: Lignes, 2009.

Foucault, Michel. Herculine Barbin dite Alexina B. Postface d'Éric Fassin. Paris: Gallimard, 2014 (nouv. éd.).

Galzy, Jeanne. Les Allongés. Paris: Gallimard, 1923.

Galzy, Jeanne. Jeunes filles en serre chaude. Paris: Gallimard, 1934.

Garréta, Anne F. Le Sphinx. Paris: Grasset, 1986.

Hall, Marguerite Radclyffe. Le Puits de solitude. Paris: Gallimard, 2005.

Havet, Mireille. Carnaval. Éd. Claire Paulhan. Paris: Éditions Claire Paulhan, 2005.

Irigaray, Luce. Le Corps-à-corps avec la mère. Montréal: Les éditions de la Pleine Lune, 1981.

Kristeva, Julia. Le Génie féminin. Tome III: Colette ou la chair du monde. Paris: Fayard, 2002.

Margueritte, Victor. La Garçonne. Paris: Petite Bibliothèque Payot, 2013.

Marks, Elaine. «Lesbian Intertextuality». Homosexualities and French Literature. Cultural Contexts / Critical Texts. Éd. George Strambolian et Elaine Marks. Ithaca-Londres: Cornell University Press, 1979. 353-377.

Maurras, Charles. Le Romantisme féminin. Allégorie du sentiment désordonné. In : L'Avenir de l'intelligence, suivi de Auguste Comte; Le Romantisme féminin, Paris: Nouvelle Librairie Nationale, 1917. 161-269.

Pelletier, Madeleine. La Femme vierge. Paris: V. Bresle, 1933.

Retaillaud-Bajac, Emmanuelle. Mireille Havet, l'enfant terrible. Paris: Grasset, 2008.

Rich, Adrienne. La Contrainte à l'hétérosexualité et autres essais. Genève: Mamamélis-Nouvelles Questions Féministes, 2010.

Robinson, Christopher. Scandal in the Ink. Male and Female Homosexuality in Twentieth-century French Literature. Londres-New York: Cassell, 1995. 
Saez Tajafuerce, Begoña. Éd. Cuerpo, memoria y representación. Judith Butler y Adriana Cavarero en diálogo. Barcelone: Icaria Editorial, 2014.

Vivien, Renée. Études et préludes. Paris: A. Lemerre, 1901.

Vivien, Renée. Une femme m'apparût. Paris: Régine Desforges, 1977.

Waelti-Walters, Jennifer. Damned Women. Lesbians in French Novels. Montréal: McGill-Queen's University Press, 2000.

Weiss, Andrea. Paris était une femme. Paris: Anatolia, 1996.

Wittig, Monique. Les Guérillères. Paris: Les Éditions de Minuit, 1969.

Wittig, Monique. Le Corps lesbien. Paris: Les Éditions de Minuit, 1973.

Wittig, Monique. La Pensée straight. Paris: Éditions Amsterdam, 2007. 